\title{
Sedimentation tank design for rural communities in the hilly regions of Nepal
}

\author{
Emilia Wisniewski \\ Department of Chemical and Biomolecular Engineering, \\ Melbourne School of Engineering, The University of Melbourne \\ emwisnie@gmail.com
}

\begin{abstract}
Mathillo Semrang in rural Nepal relies on stream sources to provide drinking water. Erosion and deforestation of local terrain produces turbid water that requires treatment before distribution. The round gravity sedimentation technology currently employed is large in footprint and cannot handle large increases in influent flow rate and silt concentrations resulting from extreme weather events. In partnership with Nepal Water for Health (NEWAH) and Engineers Without Borders Australia (EWB), this project aimed to design a highly simplified, small footprint inclined plate settler (IPS) to treat influent flow-rates ranging from 0.25 to $4 \mathrm{~L} / \mathrm{s}$. A $76 \%$ decrease in footprint was achieved by the IPS design for source flow-rates up to $4 \mathrm{~L} / \mathrm{s}$. Laboratory analysis revealed that a large inlet area along with a highly sloped floor is essential to the design which may prove problematic in the reduction of footprint in the design. Further research is needed to confirm this finding along with further collaboration with rural Nepalese communities and NEWAH.
\end{abstract}

KEYWORDS: sedimentation; clarification; Stokes' theory; inclined plate settlers.

1

\section{INTRODUCTION}

\subsection{Drinking water in Thumi VDC - Nepal}

The secure provision and distribution of drinking water is crucial in boosting socio-economical development and increasing standards of living in developing countries. Nepal ranks among the top nations in terms of fresh water potential but the majority of the population in rural areas do not have access to safe drinking water (Water Resources Management Committee, 2010). One such rural village is Mathillo Semrang in the Thumi Village Development Community (VDC) in the Western Region of Nepal.

Drinking water in Mathillo Semrang is sourced from hill and mountain streams that are subject to high sediment loads, particularly during monsoon and landslide events. Large concentrations of fine sediments in suspension result in highly turbid water sources. Deforestation and land degradation are key contributors to high sediment inflows (Julien \& Shah, 2005).

Nepal Water for Health (NEWAH) is the national Nepalese non-government organisation established to address the water and sanitation (WASH) needs of the rural and remote communities of Nepal (Nepal
Water for Health, 2011b). Many NEWAH WASH programs involve the upgrade or installation of water supply systems from source to tap. In the hilly western regions of Nepal, where sources are turbid, NEWAH and local workers install sedimentation facilities prior to the distribution of water to villages. These sedimentation facilities consist of round sedimentation tanks employing gravity sedimentation. However, it has been identified by NEWAH that in many cases there is insufficient space for the existing technology. And where the technology is of an appropriate size, it is not robust enough to cater for extreme weather conditions that result in a rapid increase in volumetric flow, such as monsoonal rainfall or landslides.

\subsection{Description of current design}

The current system employed by NEWAH is a series of five round "Ferrocement Water Filter" tanks. These tanks consist of a round clarification device with a rectangular valve box attached at the rear (Figure 1 and Figures $2 a / b$ ). The tank is doughnut in shape and contains two settling basins. The influent water enters the central basin where it is allowed to settle. The settled particles collect at the base of the vessel via a sloping floor. The clarified effluent in the central 
basin then enters the outer basin through a transfer pipe. This water travels in a clockwise direction to settle; this clockwise movement reduces the potential for short-circuiting in the system. The clarified effluent in this outer basin leaves the system through the outlet pipe and travels straight to distribution. The settled particles are removed from the system via a "washout cycle". This involves entering the valve box via the manhole. The valve servicing the outlet pipe is closed to allow the opening of the washout pipes. The normal operation of the system then allows the accumulated settled particles to be flushed from the system.

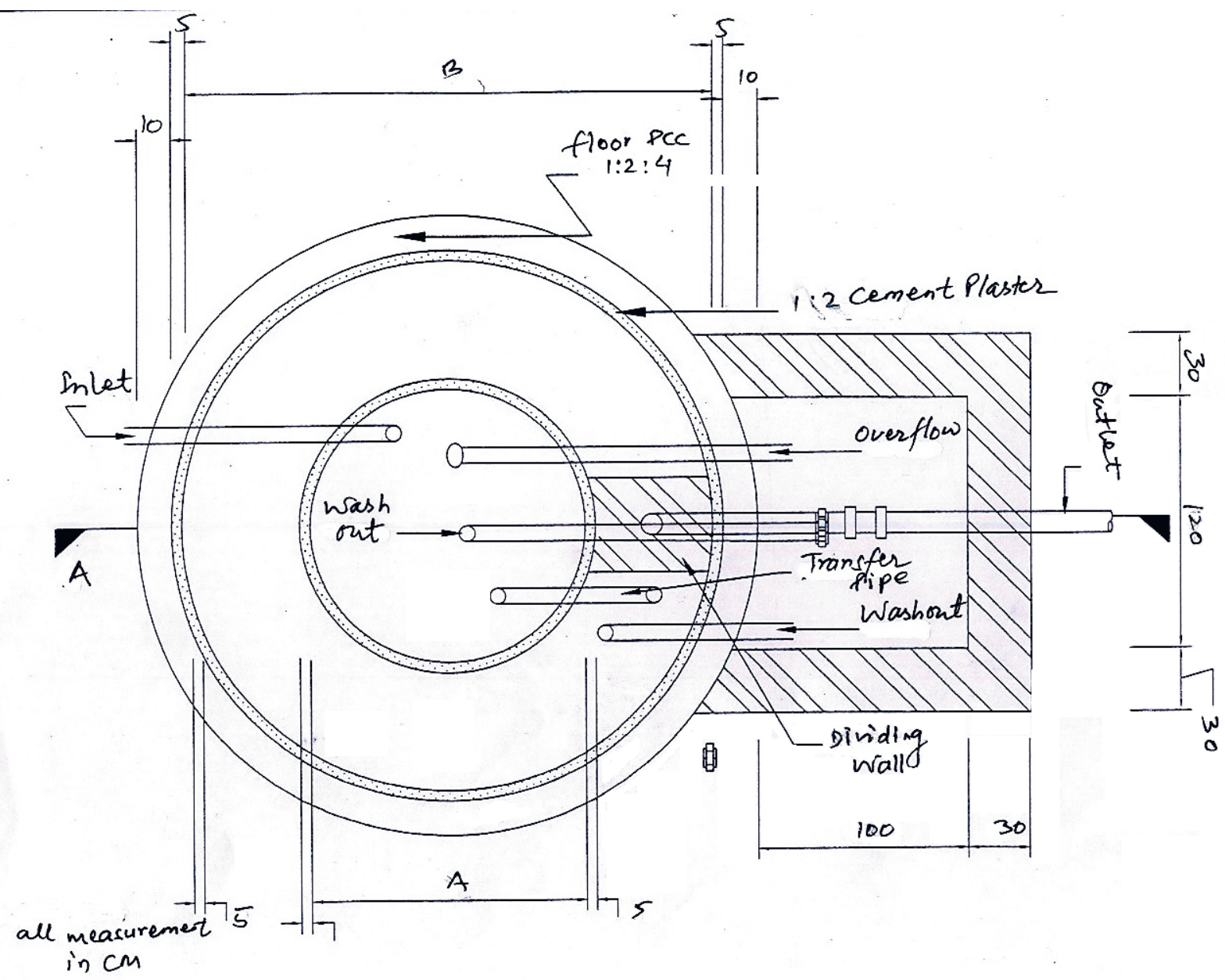

Figure 1: Top view of the current round sedimentation technology showing the two settling chambers and valve box with their corresponding piping (Nepal Water for Health, 2012).
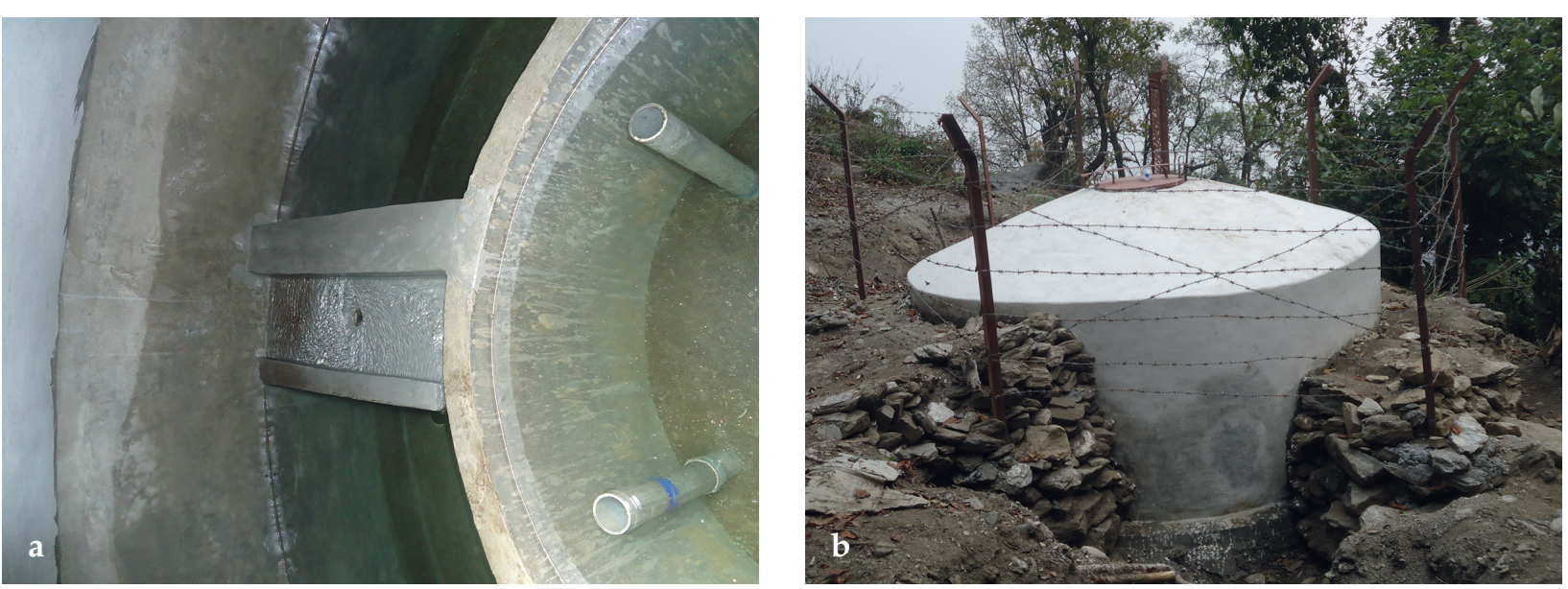

Figures $2 \mathrm{a} / \mathrm{b}$ : $\quad$ a: View of the inside of the round sedimentation tank showing the two separate settling chambers and transfer piping. b: View of the round sedimentation technology as currently constructed in Nepal showing the stone foundation and manhole covering. 


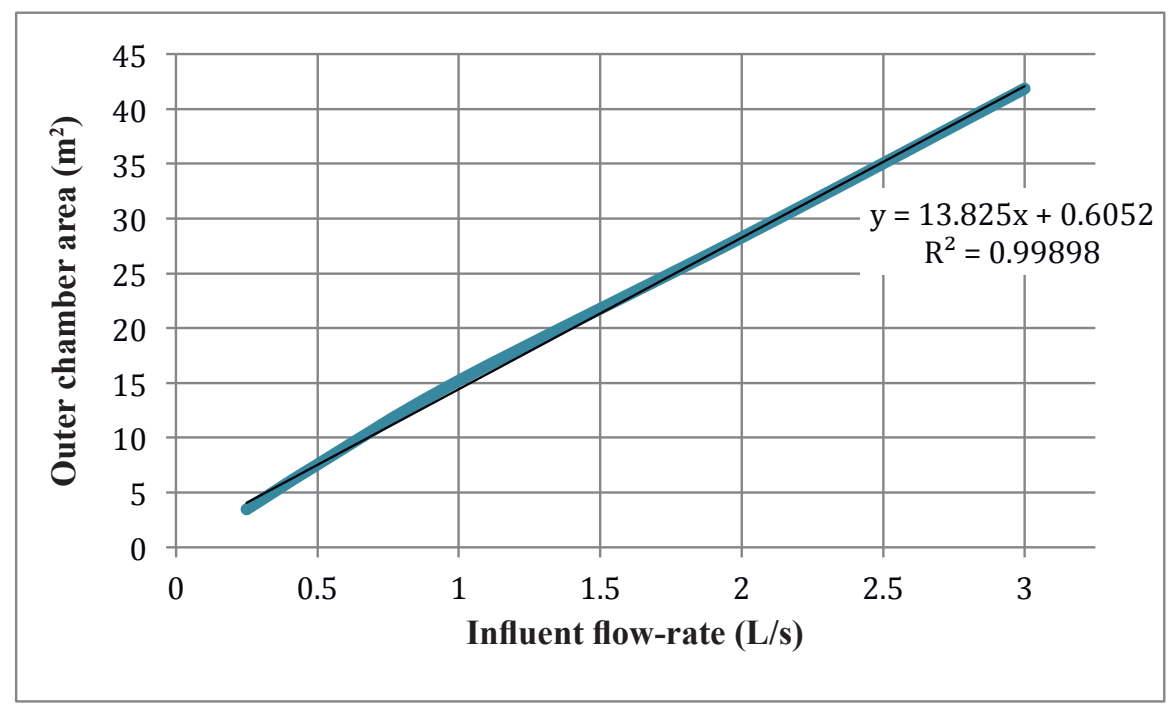

Figure 3: Cross-sectional area of current tank design with respect to the influent flow-rate.

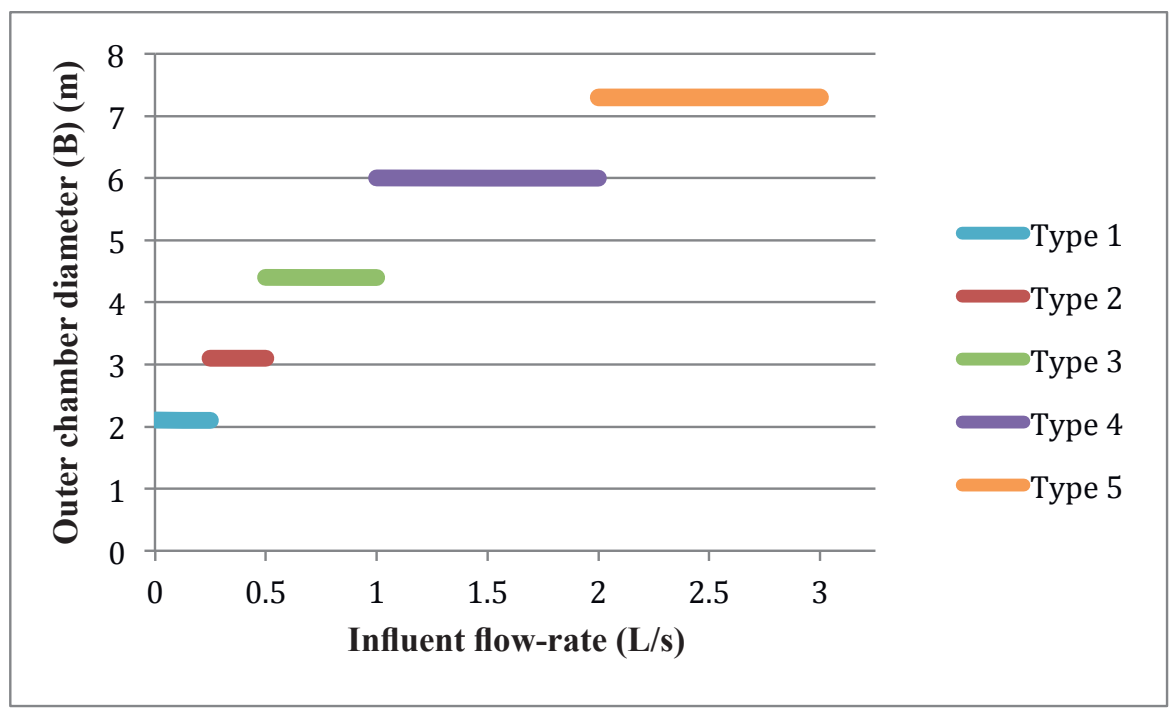

Figure 4: Variation in the diameter of the current technology with respect to the influent flow-rate.

The round tank design is a simple and cost effective technology consisting of basic building materials that are locally sourced. The two round chambers and the rectangular valve box are all constructed using Ferrocement. Simple pipe and valve fittings are included to channel the influent and effluent through the system.

The tank designs vary in cross-sectional area to suit the influent flow rate to the system (Figure 3 ). Figure 4 provides the operational range of the five Ferrocement water tanks currently in use by NEWAH. The tanks cover flow-ranges from $0.25 \mathrm{~L} / \mathrm{s}$ to $3 \mathrm{~L} / \mathrm{s}$. Flow-rates greater than $3 \mathrm{~L} / \mathrm{s}$ require the design of a complex sedimentation tank. Mathillo Semrang has a maximum stream source flow-rate of $0.32 \mathrm{~L} / \mathrm{s}$ and currently employs the use of the Type 2 tank with a maximum area of $7.55 \mathrm{~m}^{2}$ or a tank diameter of $3.1 \mathrm{~m}$.

\subsection{Current design methodology}

Although it is not wholly certain where the specific design methodology for the round technology originates, discussion with NEWAH suggests that the basic design principles, drawings and construction techniques have been adapted from a Nepalese NGO published text entitled "Rural Gravity Flow Water Systems" (Neku \& Hillman, 1996). This text deals with rural water infrastructure design in a global context and makes no specific mention of the design considerations necessary for rural Nepal, nor the effect of conditions such as extreme weather events may have on the design process.

Although the text does not detail specifically, it is suggested that the cross-sectional area of the tank is calculated using the principal of Stokes' Law. NEWAH documentation states that the crosssectional area calculation of the NEWAH design 
is based on a silt particle diameter of 10 microns (Nepal Water for Health, 2011a). A basic calculation (Appendix 1) using the design dimensions provided by NEWAH provides confirmation of both the use of a 10 micron particle diameter and the use of Stokes' Law for design.

\subsection{Limitations of current design}

Observational evidence from NEWAH suggests that the current design is considered to be an appropriate low-cost method of water treatment, provided the influent flow-rate to the system is constant. The limitation inherent in such a design is that it provides little flexibility for the design to handle shock increases in flow-rate greater than the design flow-rate.

Although there is no data to cite the difference between flow-rates during normal rainfall and extreme weather events, taking a reasonable figure such as $0.64 \mathrm{~L} / \mathrm{s}$ (double the average maximum flow-rate experienced in Mathillo Semrang) would necessitate the construction of a 'Type 3' tank which requires an increase in diameter of $42 \%$ (3.1 $\mathrm{m}$ to 4.4 $\mathrm{m})$ and a corresponding increase in area of $101.5 \%$ $\left(7.55 \mathrm{~m}^{2}\right.$ to $\left.15.2 \mathrm{~m}^{2}\right)$. Therefore, a shock increase in flow-rate due to an extreme weather event is not catered for the in current design. Due to space limitations it is also sometimes difficult to build overly large tanks to cater for flows that are larger than the design flow-rate. Therefore, it is necessary to consider an alternative technology that would provide similar water treatment capabilities within a smaller land footprint.

\subsection{Criteria for new tank design}

To remain a viable design alongside the already established technology, the proposed design needs to meet the following criteria:

- The potential to produce water of equal or greater quality than that currently produced by the existing technology.

- Low in cost with respect to both construction and operation.

- Easy to construct and operate on steep gradients.

- Able to withstand extreme weather conditions, i.e. monsoonal rainfall and land slides.

- Small enough to suit the Nepalese water supply system.

- Requiring minimal or no machinery to construct and no electricity to operate.

\subsection{Possible solution - inclined settling}

Conventional gravity sedimentation tank design insists that the cross-sectional area of the design must allow for the adequate settling of the particles contained in the solution, given a known influent flow-rate. This maximum speed is calculated using a simple principle called Stokes' Law (Appendix 1). Although this approach is adequate where space is unlimited and flow-rate conditions are constant, where space restrictions are necessary or the rate of particle settling is hindered, such as during increases influent water turbidity due to landslide events or monsoonal conditions, the traditional design approach is inadequate.

As the physical area of the tank cannot be adjusted due to space restriction, the other solution possible is to increase the settling rate of the particles. This approach both minimises the cross-sectional area necessary for design whilst still allowing for adequate particle settling.

\subsubsection{Boycott Effect}

The Boycott Effect describes the increase in particle settling rate due to the presence of an inclined surface. This phenomenon was first described by Boycott in 1920 with the discovery that if "defribinated blood is put to stand in narrow tubes, the corpuscles sediment a good deal faster if the tube is inclined than when it is vertical" (Boycott, 1920).

The increase in settling rate can be described by imagining a settling particle within an infinite quiescent medium in a container with vertical walls. The particle must travel through the medium until it reaches the bottom surface of the container. But if the particle is inside a vessel containing an inclined surface, the particle has the opportunity to make contact with a surface and slide down to the bottom of the container without having traversed the height of the entire container. The increase in particle settling rate can therefore be seen as a decrease in settling distance (Demir, 1995) and an increase in the surface area available for settling (Davis \& Acrivos, 1985).

\subsubsection{Inclined Plate Settlers}

Inclined plate settlers (Figure 5) are high rate sedimentation devices consisting of a series of inclined parallel plates forming channels (plate stack) into which turbid waters can be fed for settling. The plate stack is installed between an inlet and outlet channel (Leung \& Probstein, 1983).

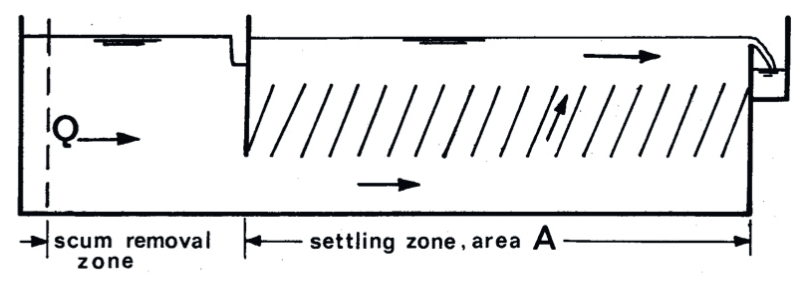

Figure 5: Cross-section of an inclined plate settler (Huisman, 1986).

Water enters through the inlet and flows through the channels created by the plate stack to the outlet area 
where the effluent is collected in the outlet chamber (Foellmi \& Bryant, n.d.; Leung \& Probstein, 1983). As the water flows through the plate stack channels, the particles settle onto the downward facing walls of the plates and slide down to the bottom of the settler (Davis et al, 1989).

IPSs possess the ability to settle very fine suspended particles at a high rate. The settler capacity per unit volume can be made large without substantial increase in footprint. The ratio of floor area needed for conventional sedimentation basins to the floor area for IPS designs can range from 8:1 to 10:1 (Foellmi \& Bryant, n.d.).

Industrial application of inclined plate settlers has been shown to be very effective in the optimisation of particle settling. Current designs allow for very compact design with very small footprints, as can be seen in Figure 6. The operation of the industrial IPS is analogous to the basic description presented above, although typically a variety of patented design features included in the Nordic Water unit, such as a flow control system and sediment thickener (Figure 6), allows for the unit to maintain very high reliability and efficiency (Nordic Water Products, 2013a).

Although providing an elegant solution to the need for a small footprint treatment technology in Nepal, there are a variety of issues that do not allow the direct integration of an industrial IPS:

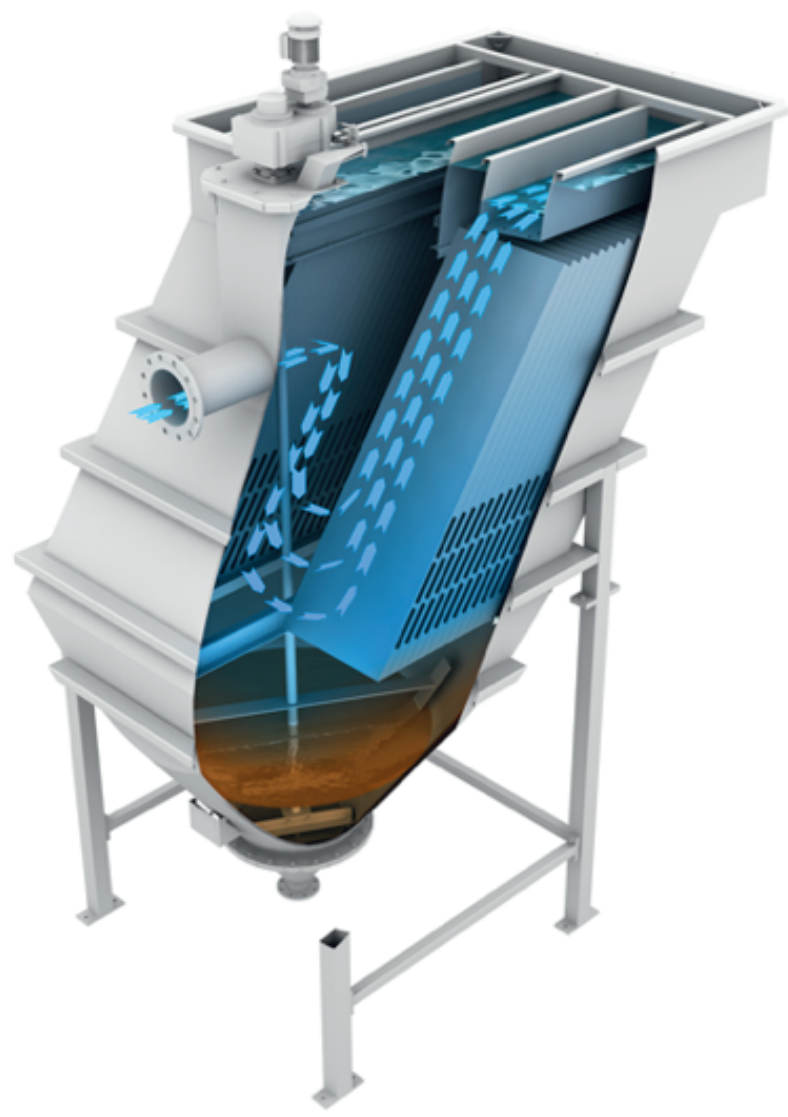

Figure 6: Industry standard inclined plate settler (Nordic Water Products, 2013b).
- The design is complex and requires extensive expert knowledge and design expertise.

- The main materials used for the design (i.e. steel/ stainless steel) are costly and are unlikely to be available in rural Nepal.

- The construction of the system is unable to be performed using manual unskilled labour.

- The system is designed as a parallelepiped in order to minimise any area loss and minimise footprint. This necessitates the construction of rigorous reinforcement to stabilise the unit and makes it less conducive to construction and operation on sloped gradients.

- The plates included in the IPS are usually very thin and spaced very close together. They are also fixed to the walls of the system. This limits the ability of the unit to be cleaned with methods other than high-pressure water or air systems and also poses a construction difficultly.

- The design of the system often incorporates the use of electricity, particularly in the case of pumps, flow control units and stirrers for thickening sludge etc.

\subsection{Project aim}

Although an industrial IPS may be inadequate to adapt for the use of rural Nepal, the basic design principle present in this design is highly useful. The aim of this project was therefore to design a highly simplified IPS that harnessed the inclined settling principle whilst still adhering to the design conditions set forth by NEWAH (Section 1.4). As the IPS design would allow such large changes in footprint, it was decided that a single design, able to treat influent flow-rates from 0.25 to $4 \mathrm{~L} / \mathrm{s}$, would be more practical than creating five separate designs corresponding to different influent flow-rate ranges. This would give the design added robustness to handle extreme events whilst still maintaining a relatively small footprint.

\section{INCLINED PLATE SETTLER DESIGN}

The approach settled upon in the construction of the IPS design was a basic rectangular cement tank that houses a simple inclined plate bundle (Figure 7). The tank structure comprises of an inlet zone, a plate bundle area, an outlet zone and a sloped floor leading to a washout pipe.

The tank will sit on a foundation of stone and may be dug into the ground if necessary. The outer walls and floors will be ferrocement with the use of adequate steel reinforcement where appropriate. A corrugated iron roof will protect the system from the weather. Any additional accessories necessary for the operation of the unit, such as valves and piping, will remain the same specification as is currently utilised. 


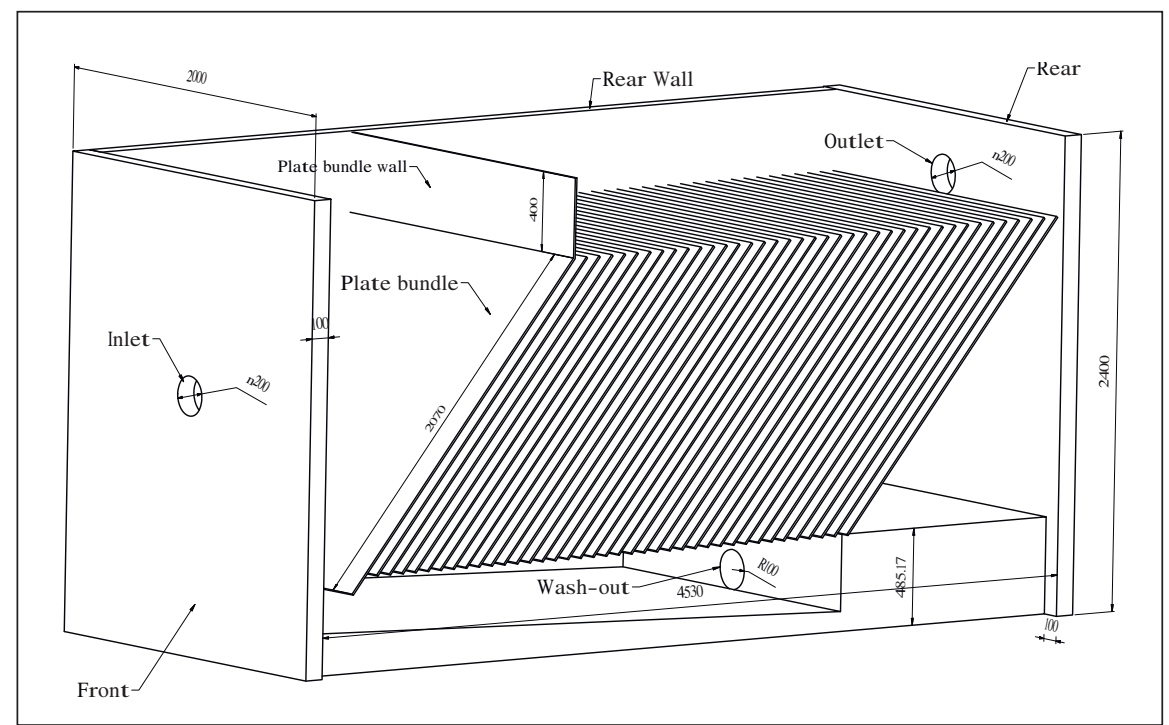

Figure 7: Isometric cross section of full-scale IPS design.

In order to ensure the IPS design remains a viable construction in competition with the current round tank design, care has been taken to ensure that the building materials and construction methods align as closely with the current methods as possible.

\subsection{Inclined plate bundle}

The area most responsible for adequate particle settling is the inclined plate bundle area. This area consists of a series of closely spaced plates attached to the left and right walls of the structure. The crosssectional area of this structure is the most important variable for calculation and dictates the overall footprint of the design. The cross-sectional area was determined by examining the physics associated with inclined particle settling and the consideration of a geometrical principle developed by Huisman (1986).

\subsubsection{Huisman's analysis}

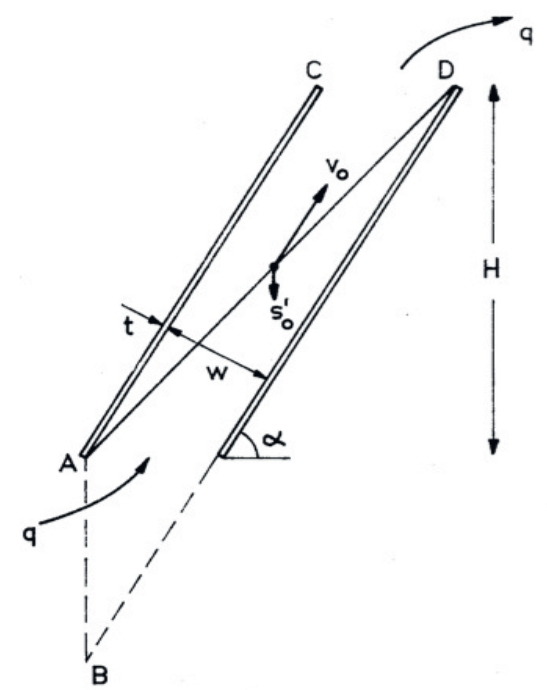

Figure 8: Schematic of settling particle between two inclined plates.
The geometric principle developed by Huisman shows the correlation between specific plate parameters and their effect on the increase in particle settling rate. As can be seen in Figure 8, the behaviour of a particle travelling in the path AC between two inclined plates can be subdivided into two separate behaviours: (a) the particular travelling linearly with velocity $\left(\mathrm{V}_{\mathrm{o}}\right)$ in the direction of water flow or $\mathrm{BC}$ and $(\mathrm{b})$ the particle settling due to gravity $\left(\mathrm{S}_{\mathrm{o}}^{\prime}\right)$ or $\mathrm{AB}$. A geometrical principle relation the distances $\mathrm{BC}$ and $\mathrm{AB}$ can then be derived which includes the parameters of the plate height bundle $(H)$, the plate thickness $(t)$, the angle of inclination $(\alpha)$, the plate spacing $(w)$.

The selection of the plate parameters depends on the design conditions. Once chosen, the 'enhanced' or 'improved' settling rate due to the inclined conditions can be described by Equation 1:

$S_{o}=S_{o}^{\prime} \frac{H \cos \propto}{w+t}$

where:

$S_{0}=$ enhanced settling rate due to inclined conditions $(\mathrm{m} / \mathrm{s})$

$S^{\prime}{ }_{0}=$ particle settling rate due to gravity (calculated from Stokes' Law) (m/s)

The overflow parameter $(U)$ is the crucial parameter in design. It is expressed as a rate of flow per unit area $\left(\mathrm{m}^{3} / \mathrm{m}^{2} \mathrm{~s}\right)($ Demir, 1995) and is generally chosen to be half of the value of the Stokes' settling rate or as in this case, half the value of the enhanced settling rate.

Once the overflow rate is obtained, the required cross-sectional area $\left(\mathrm{m}^{2}\right)$ (Equation 2) can be obtained by dividing the design influent flow-rate $(\mathrm{Q})$ by the enhanced particle settling rate. 
$A=\frac{Q}{U}$

\subsubsection{Plate bundle dimensions}

A summary of the design parameters chosen for the full-scale IPS plate scale design is listed in Table 1.

The height of the plate bundle $(H)$ was considered to be the most the parameter in the design of the plate bundle, as its height would determine the resulting height of the remaining tank structure. It was necessary for this height to be less than $2 \mathrm{~m}$ as the remaining tank structure (i.e. walls and roof) needed to be at a height that intuitively considered safe for manual construction (i.e. $2 \mathrm{~m}$ ). A plate bundle height of $1.5 \mathrm{~m}$ was chosen for the design.

A plate thickness of $0.01 \mathrm{~m}$ was chosen to allow structural integrity when inserting and removing the plates from between the tank walls. This differs to the industrial application of the IPS where extremely thin and fixed plates are used in the design. This generally limits the ability of the units to be cleaned without the use of high-pressure water or air applications. The decision to create thick plates allows for their removal from the tank structure, and the ability to clean the plates using ordinary cleaning utensils and low-pressure water application.

A plate spacing $(w)$ of $0.05 \mathrm{~m}$ was chosen to allow the ease of plate removal and insertion into the system. Industry standards generally calls for plate spacing with the region of 25 to $50 \mathrm{~mm}$ in order to minimise the resulting cross-sectional area of the plate bundle (McKean et al, 2010). But as the plates are fixed to the walls of the units, there is no need to remove the plates and the plate spacing can be minimal. A plate spacing of $0.05 \mathrm{~m}$ was chosen in order to allow for the removal of the plates for cleaning whilst still maintaining a reasonable distance to allow for adequate particle settling.

An inclination angle $(\alpha)$ of $50^{\circ}$ was chosen as a reasonable angle of inclination given the resulting cross-sectional area yielded by the Huisman principle, i.e. $5.1 \mathrm{~m}^{2}$. Sensitivity analysis conducted established that the angle of plate inclination was one the most sensitive parameters to increases in values, even with relatively small changes in angle between 10 to $30 \%$ resulting in the increase in cross-sectional area from 11 to $43 \%$ (Wisniewski, 2012). An angle of $50^{\circ}$ is also said to allow for effective self plate cleaning and the minimisation of particle entrainment (Culp et al, 1968; Shamim \& Wais, 1980).

Given the calculation of the plate bundle crosssectional area $\left(5.1 \mathrm{~m}^{2}\right)$, a reasonable plate width was chosen that would be deemed suitable to construct easily using manual labour. A figure of $1.9 \mathrm{~m}$ was chosen as it resulted in a plate bundle length less than $3 \mathrm{~m}$. Although both the plate bundle length and width are values that are ordinarily inconsequential, the maximum potential value of these parameters was considered in light of the construction practices that would be needed to construct the tank and the resulting volume of ferrocement necessary for construction.

\subsubsection{Plate Bundle Construction Materials}

The plates should ideally be constructed from sheet metal such as stainless steel, plastic (polyethylene, ABS or similar) or even a marine ply (Schulz \& Okun, 1984) but these materials are currently unavailable in rural communities. An alternative suggestion would be the use of inclined plastic tubing or an ABS hexagonal matrix instead of a plate design. This would require the redesign of the system and may prove challenging in construction and maintenance; however, such systems are common in industry. There is need for further research and liaison with the local community members as to the most appropriate material for use.

\subsection{Inlet design}

The typical design for the inlet section of an IPS would necessitate the construction of an area separate to the plate bundle with the addition of baffles in order to increase momentum dispersion in the influent and to increase effective particle settling. In order to simplify the design as much as possible and to minimise the footprint of the technology, the addition construction of a separate inlet area was discouraged, instead the inlet area was designed to make use of the wasted volume associated with the inclination of the plate bundle. Ordinarily, this volume would be eliminated by the construction of the tank in the shape of a parallelepiped. But in the case of rural Nepal, where ease of construction and simplicity in design is an imperative, the use of this space as an inlet area provide an elegant solution to the problem of increased footprint.

In order to provide adequate momentum dispersion of the influent flow, in place of additional vertical baffles, the first plate of the plate bundle was extended

Table 1: Parameters chosen for the fullscale IPS design using the Huisman analysis.

\begin{tabular}{|l|l|}
\hline Parameter & Value \\
\hline Design influent flow-rate $(\mathrm{Q})$ & $4 \mathrm{~L} / \mathrm{s}$ \\
\hline Plate bundle height $(\mathrm{H})$ & $1.5 \mathrm{~m}$ \\
\hline Plate length $(\mathrm{L})$ & $1.96 \mathrm{~m}$ \\
\hline Plate width $(\mathrm{W})$ & $1.9 \mathrm{~m}$ \\
\hline Plate thickness $(\mathrm{t})$ & $0.01 \mathrm{~m}$ \\
\hline Plate spacing $(\mathrm{w})$ & $0.05 \mathrm{~m}$ \\
\hline Angle of inclination $(\alpha)$ & $50^{\circ}$ \\
\hline Cross-sectional area of plate stack $(\mathrm{A})$ & $5.10 \mathrm{~m}^{2}$ \\
\hline
\end{tabular}




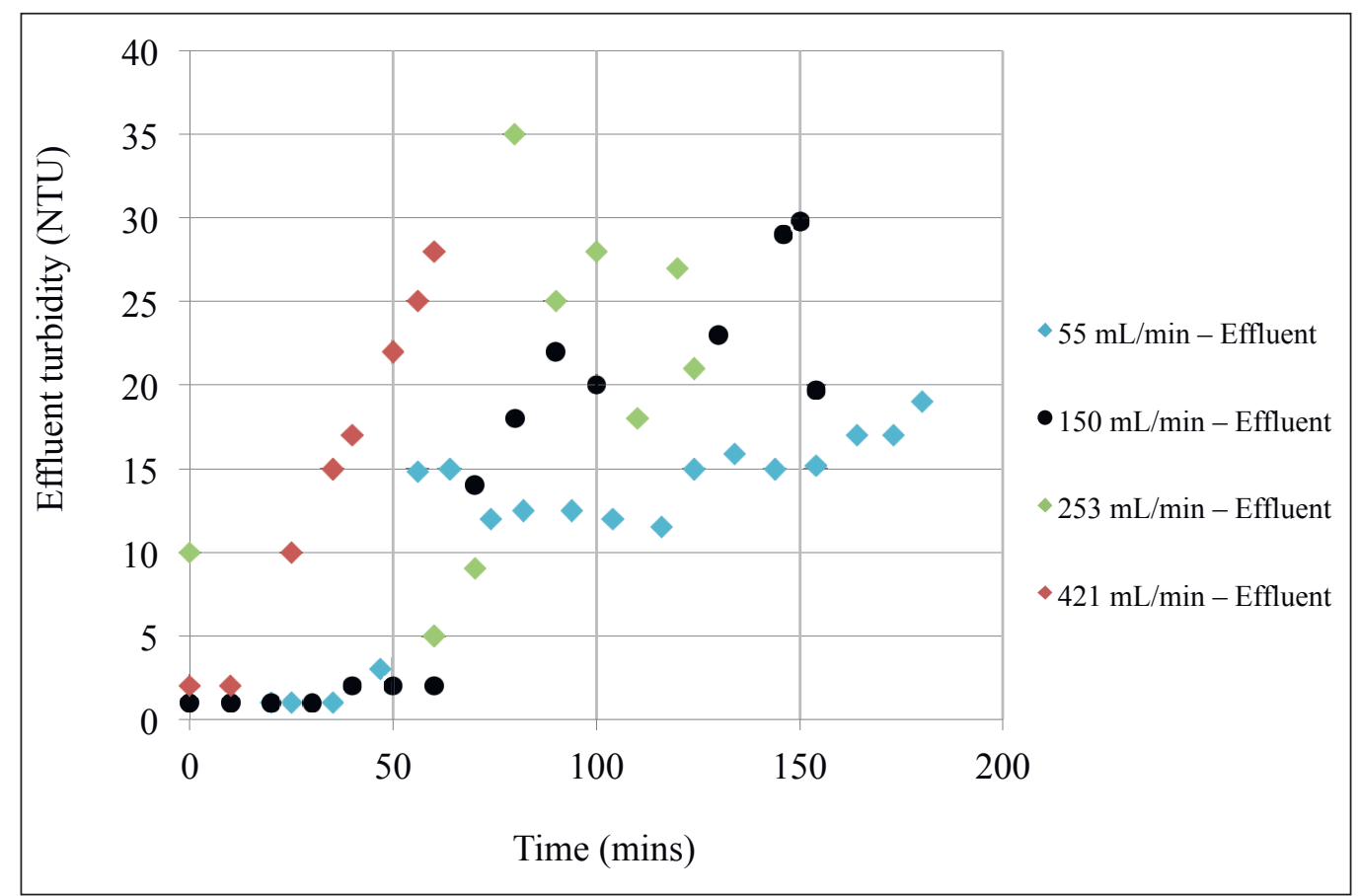

Figure 9: Effluent turbidity as a function of time for varying influent flow-rates for the lab scale model.

to create a preliminary baffle. The inclination angle of this baffle also serves to provide a medium for some inclined settling of the influent flow prior to reaching the plate bundle.

\subsection{Outlet design}

The outlet design consists of an overflow weir leading to an effluent collection basin containing a submerged outlet to prevent the carry over of scum into distribution. The depth of the collection basin is merely indicative and can be adjusted to increase or decrease the flow-rate of the effluent as necessary or to minimise the volume of water contained.

\subsection{Sludge collection design}

The floor of the tank has been constructed with a slope of $2^{\circ}$ to channel the settle particles to the washout area where they can be flushed through the washout pipe during the washout cycle. This slope angle was chosen as it minimises the vertical height of the associated tank walls to allow for adequate clearance at the bottom of the plate bundle. The sloping floor ends at a wall corresponding to the last plate in the plate bundle. It is designed to sit flush against this wall to dissuade the entrance of particle sludge into the space between the plate bundle at the outlet area.

Given the maximum particle concentration of the influent entering the tank, additional mechanical features such as the thickening devices employed in industrial design are unnecessary. Where increased particle loading is experienced, the washout pipe can simply be left open slightly to discourage the build up of sludge at the bottom of the vessel.

\subsection{Tank walls and roof}

Figure 7 indicates the dimensions of the four walls to be built to house the plate bundle. In order to ensure safe construction, the height of the walls was limited to a height of $2.4 \mathrm{~m}$. The length of the left and right tank walls allows some added room to allow for the influent flow to navigate the preliminary baffle and also concession has been made for the inclusion of an outlet zone. The length of the front and back walls was determined by the width of the plate bundle. Although mechanical design was not the main scope of this paper, the thickness of the walls was decided to be a minimum of $100 \mathrm{~mm}$, this allows for the creation of grooves in the left and right walls to accommodate the plate bundle whilst also allowing for the addition of steel rebar for structural support.

The internal structure of the tank is to be protected from the elements by a simple roof created from corrugated iron or a similar material. The roof may be attached via hinges or secured in some manner that would allow it to be easily removed to reveal the contents of the tank.

\section{LABORATORY TESTING}

A Perspex model was built to test the integrity of the full-scale design; the model could not be built to scale as scaling of the tank walls resulted in nonrealistic dimensions for the plate bundle. For ease of construction, the model did not include the overflow weir or submerged outlet or a sloping floor included leading to a washout system.

A maximum flow-rate of $421 \mathrm{~mL} / \mathrm{min}$ was calculated 
using Huisman analysis for the scaled design.

A laboratory experiment was conducted to test the ability of the scaled model to clarify solutions containing calcium carbonate particles. The influent solution was pre-prepared by settling a small amount of Omycarb 40 (calcium carbonate) in a beaker to collect the 15 micron particles needed for experimentation. The sediment was diluted with an appropriate volume of water to provide a solution with a turbidity of 20-25 NTU.

A peristaltic pump allowed the calibration of the influent to an initial $55 \mathrm{~mL} / \mathrm{min}$, or approximately $13 \%$ of the maximum flow-rate. The model tank was filled with water and a particle solution added. When the inlet chamber solution was recorded as having a turbidity of approximately 25 NTU, the effluent turbidity was measured approximately every 10 minutes to gauge operation of the device. When the effluent turbidity reached the same value as the influent, the process was stopped and the tank was emptied and cleaned.

The procedure was repeated at flow rates of 150 and $253 \mathrm{~mL} / \mathrm{min}$ (36 and $60 \%$ of the maximum flow-rate respectively) and at $421 \mathrm{~mL} / \mathrm{min}$ (maximum flowrate).

\section{$4 \quad$ RESULTS}

Figure 9 indicates that an increase in flow-rate from 13 to $60 \%$ of the maximum flow-rate $(421 \mathrm{~mL} /$ $\mathrm{min}$ ) resulted in an increase in effluent turbidity of $40 \%$. With the exception of the maximum flow-rate, the system produces reasonable effluent less than 5 NTU within the first 60 minutes of operation. After this period, a dense build up of particles was observed in the sludge collection area. This build up of particles resulted in short-circuiting of the system either through the first or last channel for the low and higher flow-rates respectively. This result was noted by an increase in effluent turbidity after the 60-minute period. This time frame may signal the point at which a washout period is necessary; there is little indication as to whether this is a viable timeframe for operation without further testing at pilot scale. Although to stem the possible frequency of the washout cycle, the washout may be designed in such a way that enables a continuous low flow discharge to ensure adequate clarification.

The experimental results also revealed the importance of the design of the inlet chamber and sloping floor. An increase in area of the inlet chamber and a steepening of the sloping floor leading to a sump would allow an increase in momentum dissipation and an increase in system performance. These changes are a challenge for the design, as the increase in the dimension of these areas results in an increase in footprint and vertical height of the system, proving difficult for construction.
If the experimental results are somewhat representative of the potential operation of the system at full-scale, it may be suggested that the simplistic nature of the design does not allow for adequate clarification, but as the laboratory model was not a perfect representative of the system, this conclusion requires further validation with the construction of a pilot scale model. Experimental testing with a wide range of feed flow-rates, particle concentrations and turbidities would be necessary to fully understand system performance.

\section{CONCLUSIONS AND RECOMMENDATIONS}

A simplified IPS was designed to combat the turbidity issues arising from stream sources in the rural hilly community of Mathillo Semrang, Nepal.

The footprint of the design is $10.5 \mathrm{~m}^{2}$ with the theoretical capacity to treat a maximum influent flow-rate of approximately $4 \mathrm{~L} / \mathrm{s}$. This is a reduction in footprint of up to $76 \%$ from the current round sedimentation design $\left(42 \mathrm{~m}^{2}\right)$ required to treat an influent flow of $4 \mathrm{~L} / \mathrm{s}$. The design allows for the construction of a single device to replace the five separate devices currently implemented. The design is gravity driven and requires no electricity to operate. Its construction is similar to that of the current design and is therefore assumed to be economically viable.

Although the theoretical basis for the design is promising, laboratory experimentation identified necessary changes in the design structure to allow for adequate sedimentation, such as increase in the slope of the floor and an increase in the area of the inlet zone to allow for adequate momentum dispersion.

The overall success of the design in handling a design influent flow-rate of $4 \mathrm{~L} / \mathrm{s}$ and the ability of the system to handle extreme flow-rate is difficult to determine without the construction of a pilot system but further bench experimentation such as particle size distribution testing of the effluent and flow modelling of the system may be useful in revealing any shortcomings of the design.

Collaboration with NEWAH and the Mathillo Semrang community is necessary to supplement the design particularly in the fields of material selection and construction.

\section{ACKNOWLEDGEMENTS}

The author would like to express thanks towards the supervisors of this project - Prof. Peter Scales (University of Melbourne) and Dani Barrington (EWB Australia / Nepal Water for Health).

Disclaimer: A version of this paper was first published in Macquarie Matrix (Volume2.2, December 2012) 


\section{REFERENCES}

Boycott, A. E. 1920. Sedimentation of blood corpuscles. Nature, 104, p. 532.

Culp, G., Hansen, S. \& Richardson, G. 1968. High rate sedimentation in water treatment works. Journal of the American Water Works Association, 681-936.

Davis, R. H. \& Acrivos, A. 1985. Sedimentation of noncollodial particles at low Reynold's numbers. Annual Review of Fluid Mechanics, 17, pp. 91-118.

Davis, R. H., Zhang, X. \& Agarwala, J. P. 1989. Particle classification for dilute suspensions using an inclined settler. Industrial \& Engineering Chemistry Research, 28, pp. 785-793.

Demir, A. 1995. Determination of settling efficiency and optimum plate angle for plated settling tanks. Water Research, 29 (2), pp. 611-616.

Foellmi, S. N. \& Bryant, H. H. n.d. Lamella Plate Settlers, Design and Operation: Two Case Histories, from http:/ / www.terrestorm.com/press_research_ Foellmi_and_Bryant.pdf

Huisman, L. 1986. Discrete Settling in Continuous Horizontal Flow Basins Sedimentation and Flotation: Mechanical Filtration, pp.38-96. Delft, The Netherlands: Delft University of Technology.

Julien, P. \& Shah, S. 2005. Sedimentation Initiatives in Developing Countries. Draft report presented to UNESCO-ISI. Colarado State University, USA.

Leung, W-F \& Probstein, R. F. 1983. Lamella and Tube Settlers. 1. Model and Operation. Industrial $\mathcal{E}$ Engineering Chemistry Process Design and Development, 22(1), 58-67.

McKean, T., Bourke, B., Mitchell, W., Caplygin, L. \& McKeown, R. 2010. Novel application of a lamella clarifier for improved primary treatment of domestic wastewater. Paper presented at the 73rd Annual Victorian Water Industry Engineers and Operators Conference, Exhibition Centre - Bendigo.

Neku, A. \& Hillman E.A. 1996. Chapter 16: Sedimentation Tank. In R. Sack \& D. Crawford Eds., Rural Gravity Flow Water Systems Design Technique and Standard Structures. Kathmandu, Nepal: UNICEF Nepal.

Nepal Water for Health. 2011a. 2.6 Sedimentation Tank Design Rural Drinking Water Supply System Policy and Procedures (3 ed.). Lothsal, Kathmandu, Nepal.

Nepal Water for Health. 2011b. Rural Drinking Water Supply System Policy and Procedures (3 ed.). Lothsal, Kathmandu, Nepal.

Nepal Water for Health. 2012. Technical drawing of the top view of the current round sedimentation technology. Kathmandu, Nepal: Nepal Water for Health.
Nordic Water Products. 2013a. Lamella Separator - for optimal sedimentation Retrieved 03/05/13, from http:/ /www.nordicwater.com/en/index.cfm/ product-groups/lamella/

Nordic Water Products. 2013b. Lamelle Separator for optimal sedimentation.

Schulz, C. R. \& Okun, D. A. 1984. Inclined tube and plate settling Surface Water Treatment of Communities in Developing Countries (pp. 139-144). London: Intermediate Technology Publications.

Shamim, A. \& Wais, M. T. 1980. Potential of tube settlers in removing raw water turbidity prior to coagulant. Aqua, 8, pp. 166-169.

Water Resources Management Committee. 2010. Water Use Master Plan - Thumi VDC Gorka. Thumi VDC, Nepal.

Wisniewski, E. 2012. Sedimentation Tank Design for Disadvantaged Rural Communities in the Hilly Regions of Nepal. Engineering Research Report. The Department of Chemical and Biomolecular Engineering. The University of Melbourne.

\section{APPENDIX 1}

\section{A1.1 Fundamental design principles of sedimentation units}

The design of the current system is based on the principle of gravity settling. Gravity settling occurs in tanks of water with large cross-sectional areas where small influent and outward flows create a state of virtual quiescence in the system. Under the influence of gravity, particles with densities higher than that of the surrounding fluid sink (sedimentation) whilst lighter particles float (Huisman, 1986). The particles present in the system are retained in a sludge layer at the bottom of the tank and the water leaves the system in a clarified state (Huisman, 1986).

The rate of the fall of the particles depends on the particle size. Large particles descend more rapidly than small particles. The size of particle can be manipulated by aggregation. This segregates the types of settling experience into two types: discrete and flocculated.

Discrete settling occurs in systems with low particle concentration where aggregation is negligible. In discrete settling, the settling rate of the particles can be calculated using Stokes' law (Equation A1).

$S_{o}^{\prime}=\frac{d^{2} \times g \times\left(\rho-\rho_{f}\right)}{18 \times \mu}$

where, $d$ is the particle diameter, $g$ is the gravitational acceleration constant $\left(\mathrm{m}^{2} / \mathrm{s}\right), \mu$ is the fluid viscosity $\left(\mathrm{Ns} / \mathrm{m}^{2}\right), \rho$ is the particle density $\left(\mathrm{kg} / \mathrm{m}^{3}\right), \rho_{f}$ is the fluid density $\left(\mathrm{kg} / \mathrm{m}^{3}\right)$ and $S^{\prime}{ }_{0}$ is the Stokes' settling rate of particle $(\mathrm{m} / \mathrm{s})$. The value of the parameters 
used for the calculation of settling rates can be seen in Table A1.

The tank dimensions of the current technology (Figure 1) provided by NEWAH are listed in Table A2. Both the apparent footprint of the technology (D) and the Stokes' settling rate of a 10 micron particle (E) were calculated using Stokes' Law (Equation A1).
The average of the settling rate was calculated to be approximately $1.08 \times 10^{-4} \mathrm{~m} / \mathrm{s}$. A comparison to the settling rates obtained by Equation $\mathrm{A} 1$ by varying the particle diameter (Table A3) confirms that an average particle size of approximately 1.08 microns was used as a basis for the calculation of the tank dimensions. This validates the use of a 10 micron particle as a basis for this project.
Table A1: Parameters used in the calculation of the settling rates seen in Table A2.

\begin{tabular}{|l|c|l|}
\hline$d(\mathrm{~m})$ & $1.00 \times 10^{-5}$ & \\
\hline$g\left(\mathrm{~m}^{2} / \mathrm{s}\right)$ & 9.81 & \\
\hline$\mu\left(\mathrm{Ns} / \mathrm{m}^{2}\right)$ & $1.00 \times 10^{-3}$ & Water at $20^{\circ} \mathrm{C}$ \\
\hline$\rho\left(\mathrm{kg} / \mathrm{m}^{3}\right)$ & 2700 & Average density of graphite particle \\
\hline$\rho_{f}\left(\mathrm{~kg} / \mathrm{m}^{3}\right)$ & 998.2 & Water at $20^{\circ} \mathrm{C}$ \\
\hline
\end{tabular}

Table A3: Variation in Stokes' settling rate with respect to particle size.

\begin{tabular}{|c|c|}
\hline $\mathbf{G}$ & $\mathbf{H}$ \\
\hline $\begin{array}{c}\text { Particle } \\
\text { diameter } \\
\text { (microns) }\end{array}$ & $\begin{array}{c}\text { Stokes' settling } \\
\text { rate (m/s) }\end{array}$ \\
\hline 1 & $9.27 \times 10^{-5}$ \\
\hline 1.01 & $9.46 \times 10^{-5}$ \\
\hline 1.02 & $9.65 \times 10^{-5}$ \\
\hline 1.03 & $9.84 \times 10^{-5}$ \\
\hline 1.05 & $1.02 \times 10^{-4}$ \\
\hline 1.08 & $1.08 \times 10^{-4}$ \\
\hline 1.20 & $1.34 \times 10^{-4}$ \\
\hline 1.50 & $2.09 \times 10^{-4}$ \\
\hline 2.0 & $3.71 \times 10^{-4}$ \\
\hline 3.0 & $8.35 \times 10^{-4}$ \\
\hline
\end{tabular}

Table A2: Dimensions and settling properties of the current round ferrocement technology established in Nepal.

\begin{tabular}{|c|c|c|c|c|c|c|}
\hline & $\mathbf{A}$ & $\mathbf{B}$ & $\mathbf{C}$ & $\mathbf{D}$ & $\mathbf{E}$ & $\mathbf{F}$ \\
\hline & $\begin{array}{c}\text { Tank } \\
\text { Design }\end{array}$ & $\begin{array}{c}\text { Influent } \\
\text { flow-rate } \\
\text { (L/s) }\end{array}$ & $\begin{array}{c}\text { Outer } \\
\text { Chamber } \\
\text { Diameter } \\
{[\mathbf{B}] \mathbf{( m )}}\end{array}$ & $\begin{array}{c}\text { Outer } \\
\text { Chamber area } \\
\left(\mathbf{m}^{2}\right)\end{array}$ & $\begin{array}{c}\text { Stokes' } \\
\text { settling } \\
\text { rate of } \\
\text { particles } \\
\text { (m/s) }\end{array}$ & $\begin{array}{c}\text { Variation } \\
\text { from } \\
\text { average } \\
\text { settling } \\
\text { rate (\%) }\end{array}$ \\
\hline & Type 1 & $\leq 0.25$ & 2.1 & 3.46 & $7.22 \times 10^{-5}$ & -49 \\
\hline & Type 2 & 0.25 to $\leq 0.5$ & 3.1 & 7.55 & $1.32 \times 10^{-4}$ & 19 \\
\hline & Type 3 & 0.5 to $\leq 1$ & 4.4 & 15.2 & $1.32 \times 10^{-4}$ & 18 \\
\hline & Type 4 & 1 to $\leq 2$ & 6 & 28.3 & $1.06 \times 10^{-4}$ & -1 \\
\hline & Type 5 & 2 to $\leq 3$ & 7.3 & 41.9 & $9.56 \times 10^{-5}$ & -13 \\
\hline AVERAGE & & & & & $1.08 \times 10^{-4}$ & \\
\hline
\end{tabular}


